\title{
Los mundos virtuales: una reflexión más allá de lo digital
}

\section{Jesus Peralta ${ }^{1}$ y \\ Alejandro Miranda² \\ Universidad Nacional Autónoma de México}

\section{Ensayo}

Material original autorizado para su primera publicación en Journal de Ciencias Sociales, Revista Académica de la Facultad de Ciencias Sociales de la Universidad de Palermo.

Recepción: 07-07-2021

Aceptación: 28-10-2021

Resumen: El desarrollo tecnológico ha posibilitado que la Internet sea un espacio que alberga nuevas realidades conocidas como mundos virtuales, los cuales se caracterizan por ser entornos simbólicos, en donde los usuarios pueden interactuar por medio de autorrepresentaciones digitales conocidas como avatares. Algunos ejemplos de estos mundos son Minecraft, Second Life, Garena Free Fire o World of Warcraft, en los que millones de personas interactúan simultáneamente gracias a las potencialidades de las tecnologías que subyacen. Pese a lo que se podría pensar, estos mundos virtuales existen desde antes de la aparición de la Internet y se articulan en diferentes juegos de rol, historias, mitos, leyendas y creencias que forman parte del acervo cultural, los cuales se caracterizaban por promover procesos de socialización y aculturación, fomentar la identidad social y la cohesión, etcétera. Al tratarse de espacios simbólicos, estos escenarios precisan de distintos artefactos culturales que permiten la interacción con ellos. En este sentido, es posible pensar que los mundos virtuales pueden prescindir de la infraestructura tecnológica, pero no de una infraestructura simbólica. De aquí que el objetivo de esta disertación teórica es analizar aquellos artefactos culturales que dan sustento y cabida a los mundos virtuales. Para ello, se plantean tres ejes analíticos: los fenómenos relacionados a la creación de nuevos mundos,

\footnotetext{
${ }^{1}$ Profesor en la Facultad de Estudios Superiores Iztacala de la Universidad Nacional Autónoma de México. Miembro del Proyecto de Investigación PsicoEducativa. Estudiante del Doctorado en Psicología Educativa. Correo electrónico: jesus.peralta@iztacala.unam.mx

2 Profesor de Carrera de tiempo completo en la Facultad de Estudios Superiores Iztacala de la Universidad Nacional Autónoma de México. Está adscrito al Sistema Universidad Abierta y Educación a Distancia Iztacala y es miembro del Proyecto de Investigación PsicoEducativa. Coordina el grupo de profesores de Comunidad de Habilidades y Aprendizaje con Tecnología (CHAT).

Correo electrónico: amiranda@iztacala.unam.mx
} 
los mecanismos que posibilitan la habitabilidad de mundos intangibles y finalmente, los instrumentos de comunicación subyacentes.

Palabras clave: mundos virtuales; artefactos culturales, creatividad, lenguaje.

\section{Virtual worlds: a reflection beyond the digital}

Abstract: Technological development has enabled the Internet to be a space that contains new realities known as virtual worlds, which are characterized by being symbolic environments, where users can interact through digital self-representations known as avatars. Some examples of these worlds are Minecraft, Second Life, Garena Free Fire, or World of Warcraft, where millions of people interact simultaneously thanks to the potential of the underlying technologies. Despite what one might think, these virtual worlds have existed since before the appearance of the Internet and are articulated in different role-playing games, stories, myths, legends, and beliefs that are part of the cultural heritage, which were characterized by promoting processes of socialization and acculturation, promoting social identity and cohesion, and so on. As they are symbolic spaces, these settings require different cultural artifacts that allow interaction with them. In this sense, it is possible to think that virtual worlds can do without technological infrastructure, but not a symbolic infrastructure. Hence, the objective of this theoretical dissertation is to analyze those cultural artifacts that support virtual worlds. For this, three analytical axes are proposed: the phenomena related to the creation of new worlds, the mechanisms that enable the habitability of intangible worlds, and finally the underlying communication instruments.

Keywords: virtual worlds; cultural artifacts, creativity, language.

\section{Introducción}

Desde la década de los años 60, el proyecto experimental ARPANET, representó el primer antecedente del internet. El desarrollo tecnológico que se ha suscitado desde aquel precedente favoreció la aparición en 1995 de la Internet comercial que se conoce actualmente (Díaz, 2016). Veinticinco años después, esta mediación se ha convertido en una parte indispensable de la vida cotidiana para todas las personas.

La Internet es una red universal interconectada de comunicaciones que se soporta de diferentes medios como el audio, el video y el texto electrónico, permitiendo que las formas de comunicación que antes estaban separadas ahora converjan y se superpongan entre sí. La Internet se caracteriza por difuminar la distinción entre comunicación interpersonal y masiva; entre la comunicación privada y la comunicación pública (Sparrow, 2012). 
De acuerdo con Timokhina y Emelianenko (2018), el funcionamiento de las comunicaciones en internet tiene una condición importante: la organización de los sistemas humanos en un espacio virtual. El término "virtualidad" se utilizaba anteriormente solo para referir a aquella práctica social o discursiva que permitía al ser humano interpretar todo aquello que es real. No obstante, la aparición de la Internet transformó la acepción del término, para referirse a un entorno simbólico apoyado de herramientas informáticas que lo hacen habitable.

Los mundos virtuales son la manifestación perfecta de la virtualidad. Para el creador de uno de los mundos virtuales más populares (Second Life), estos entornos son una reacción a la sensación de soledad que muchos usuarios experimentan en la navegación dentro de la Red (Sparrow, 2012). Se sabe que el ciberespacio está repleto de personas, pero cuando un usuario visita un sitio web, éste no percibe la existencia de nadie más que él, ya que el entorno gráfico no provee de las representaciones necesarias para sentir la presencia de los demás usuarios. De aquí que, un mundo virtual, bajo esta acepción, refiera a un entorno que contextualiza los encuentros sociales (Sparrow, 2012).

La aparición de los mundos virtuales en la Internet ha trastocado diferentes esferas sociales. Por mencionar algunos campos, Adams (2014) indica que el surgimiento de entornos virtuales ha llevado a la reconceptualización de la comunicación humana.

A través de un análisis histórico de los diferentes medios de comunicación que el ser humano ha implementado para su interacción con otros, el autor plantea que la comunicación, bajo cualquier medio, se caracteriza por una "fragmentación de la conciencia" que permite a personas dispersas tener contacto directo (Adams, 2014).

De igual modo, la actividad económica se ha transformado con la presencia de los mundos virtuales. Lehdonvitra (2014) utiliza el concepto de bienes virtuales para referirse a objetos digitales que son comprados e intercambiados en la Internet. El autor plantea que estos bienes se han convertido en una forma popular de gastar dinero en juegos para teléfonos móviles y dispositivos como tabletas.

Un caso consolidado de este fenómeno se encuentra en Second Life, que se caracteriza por ofrecer una comunidad y un mercado en línea altamente sofisticados como resultado de un largo proceso de desarrollo, tanto a nivel demográfico, como a nivel económico. En dicho mundo virtual, existe una gran variedad de bienes a la venta disponibles para el consumo del avatar, los cuales incluyen ropa, productos electrónicos, propiedades, casas, muebles, vehículos y cualquier otra cosa que se pueda imaginar y crear mediante software (Martin, 2008). 
Esta clase de transacciones, ha dado lugar a nuevas realidades económicas que se caracterizan por el intercambio par a par. Esta nueva clase de economía colaborativa se basa en plataformas conocidas como Blockchain, que permiten a sus usuarios vender activos virtuales o productos multimedia entre sí, sin la necesidad de tener que acceder a un gestor de activos centralizado (Coyne, 2018).

Dentro de mundos virtuales como Second life, esta tecnología ha permitido a los usuarios controlar y administrar sus bienes virtuales con independencia de los operadores del mundo virtual.

La comunicación y la economía, son sólo algunos casos que demuestran las importantes implicaciones de los mundos virtuales en la vida social. Pero para poder comprender por qué la virtualidad ha tenido tal impacto, resulta necesario detenerse a analizar los fundamentos que sustentan esta clase de entornos simbólicos.

Gran parte de la literatura plantea que la principal fundamentación de los mundos virtuales en la Internet subyace a la infraestructura digital basada en sistemas de telecomunicación, hardware y software que posibilitan la creación, la mediación y representación de dichos entornos (Greenlaw y Hepp 2001).

Sin embargo, la existencia de los mundos virtuales precede a la aparición y consolidación de dicha herramienta contemporánea, cuando las personas solían representar escenarios intangibles a través de historias, leyendas, mitos, juegos de rol, etcétera. Por lo tanto, si los mundos virtuales existen desde antes de la creación de la Internet, es factible pensar que estos se sostienen por medio de otros artefactos culturales como el lenguaje mismo.

De ninguna manera se pretende despreciar el enorme papel que juegan las tecnologías contemporáneas como posibilitadoras de los fenómenos que ocurren en la Internet. En realidad, algunos otros posicionamientos rescatan la dimensión social y plantean que los entornos virtuales en la Red, si bien tienen un sustento tecnológico primordial, su importancia radica en el valor social detrás de ellos, pues representan un principal medio de comunicación, interacción y organización social (Castells, 2000).

En lo que respecta a este ensayo, el principal objetivo no es ampliar los análisis ya existentes de los fenómenos psicológicos, sociales o culturales de estos entornos, sino analizar los artefactos culturales que dan sustento y cabida a los mundos virtuales, pues como se expondrá más adelante, los mundos virtuales no son el resultado del desarrollo en las telecomunicaciones, sino se trata de fenómenos sociales que son tan antiguos como la civilización misma. 
De aquí que, los mundos virtuales en la Internet son sólo una variación del basto espectro de mundos simbólicos, en donde la instrumentación psicológica, social y cultural es fundamental para su existencia. En este sentido, los mundos virtuales no sólo existen en la Internet, sino en otras prácticas narrativas tales como juegos de rol, mitos, leyendas, historias, etcétera.

\section{Argumentación teórica}

\section{1. ¿Cómo entender la virtualidad?}

Hablar sobre los mundos virtuales, conlleva a reflexionar primero sobre la concepción de la virtualidad. Garner y Grimshaw (2014) proponen un interesante análisis respecto a la concepción de dicho concepto. Ellos establecen que, desde lo popular, la virtualidad tiene un significado que representa lo que es capaz de estar "cerca" o ser "auténtico", donde se intenta aproximar a las personas a experiencias lo más parecidas a la realidad.

Retomar dichas definiciones anularía cualquier esfuerzo por concebir la realidad más allá de lo concreto, de aquello que se puede ver, sentir o percibir con los sentidos, ignorando por completo aquellos fenómenos de la realidad que son intangibles, pero que el individuo experimenta, tales como los significados o las ideas.

Al respecto, Brey (2014) analiza la virtualidad en términos ontológicos. Por mucho tiempo se pensó que lo real era aquello que tenía una materialidad, sin embargo, hay objetos que existen y que no por ello ocupan un lugar físico; incluso existen objetos que pueden tener una composición material, pero que no son lo que aparentan ser.

El famoso pintor surrealista René Magritte hace evidente este planteamiento en una de sus principales obras plásticas titulada Ceci n'est pas une pipe (esto no es una pipa). Dicha pintura es básicamente la representación de una pipa que contiene la frase con la que se bautiza esta obra. El pintor niega lo evidente: lo que está representado en el lienzo (la pipa), no es realmente lo que parece. En términos generales, Magritte evidencia que la realidad concreta se encuentra separada de toda representación simbólica, iconográfica o pictórica.

Brey (2014) cuestiona el principio de la materialidad como criterio definitorio de la realidad, debido a que, si bien la virtualidad no tiene un referente físico, posee otras cualidades que la hacen real, tales como su estructura unificada y estable y la capacidad que tiene como un espacio de interacción entre seres humanos y objetos virtuales a través de interfaces computacionales.

En la virtualidad dentro de la Internet, el ser humano es capaz de interactuar con objetos digitales. Esta interacción eleva a dichos objetos a la categoría de artefactos, dado que son capaces de mediar la actividad humana dentro de los entornos virtuales. Tómese 
como ejemplo la lupa virtual que aparece en un editor de imagen, la cual le permite, a quien opera sobre el programa, realizar una acción en concreto, que consiste en acercar o alejar la imagen que se está editando. Como se aprecia en este ejemplo, la lupa es una herramienta mediadora entre el sujeto y el propósito de la acción.

En síntesis, la virtualidad se puede definir como una realidad que existe simbólicamente que, dada su intangibilidad, se requiere de ciertas mediaciones para poder operar sobre ella. A partir de esta definición, se puede afirmar que en la Internet existen realidades intangibles conocidas como mundos virtuales, a los que solo es posible acceder a través de dispositivos computacionales. En el siguiente apartado se analizarán algunas características de esta clase de fenómenos.

\subsection{Una inmersión teórica a los mundos virtuales de la Internet}

En la Internet, existen diferentes mundos virtuales tales como Fortnite, World of Warcraft, Runescape, Entropia Universe, Second Life, OpenSim, Minecraft, Animal Crossing (Minocha y Hardy, 2016). El desarrollo tecnológico ha posibilitado que la Red sea un espacio que alberga mundos virtuales que poseen características específicas.

En la Conferencia State of the Net del 2008, un grupo de expertos analizó la pluralidad de los mundos virtuales con la finalidad de generar contextos apropiados para la investigación futura. En el evento, llegaron a la conclusión de que los mundos virtuales son herramientas de Internet en 3D, en donde las personas pueden interactuar por medio de avatares. Estos entornos pueden dividirse en dos tipos: mundos virtuales abiertos, que se distinguen por ser extensiones del mundo real como Second Life, en donde los usuarios tienen la capacidad de crear cualquier cosa dentro de sus límites; y mundos virtuales cerrados, que se diferencian por delegar su creación exclusivamente a los desarrolladores y no a los usuarios (McGill, 2008).

Por otra parte, los mundos virtuales se caracterizan por ser entornos intangibles que presentan los siguientes atributos: (1) autorrepresentación mediante avatares; (2) existencia persistente; (3) sincronía; (4) configuración en red; y (5) mediación por cómputo (Bell, 2008).

La posibilidad de acción que tienen los usuarios a través de un avatar es una característica central. Un avatar es una representación virtual que posee la capacidad de agencia; este atributo lo distingue de otros fenómenos que se encuentran en la Internet (Zhang et al. 2017).

Por ejemplo, Fortnite es un mundo virtual creado por la empresa Epic Games, que destaca por una modalidad de juego de batalla masiva en tiempo real. En este mundo virtual, decenas de usuarios se enfrentan entre sí en una isla con la finalidad de ser el último en sobrevivir. Las acciones que se llevan a cabo para este cometido, están mediadas por 
personajes conocidos entre la comunidad como skins. Un usuario tiene la posibilidad de tener distintos skins que puede intercambiar en las diferentes campañas en las que participa (Narea, 2019).

En cambio, un usuario de una red social como Facebook no puede utilizar su perfil para dar "me gusta" a la foto de un amigo. La principal diferencia entre uno y otro es que el usuario controla las acciones de su avatar, pero es este último el que realiza la acción en el entorno virtual. En las redes sociales, el usuario es quien actúa sobre la interfaz gráfica sin ninguna mediación de por medio.

Una característica que Snodgrass (2016) identifica de los mundos virtuales en la Red, es su alta capacidad de subsistir independientemente de cualquier jugador o usuario, lo que denomina existencia persistente. Los usuarios pueden desconectarse, pero siempre habrá otros jugadores compitiendo, interactuando, modificando las condiciones del entorno virtual, etcétera. A diferencia de muchos videojuegos como Snow-Bross, Resident Evil, Street Fighter, o cualquier otro de su clase, en los mundos virtuales es imposible pausar la cadena de experiencias suscitadas, por lo que éstos persisten independientemente del usuario.

La sincronía es otra cualidad de los mundos virtuales en el ciberespacio. De acuerdo con Bell (2008), la noción del tiempo compartido que se experimenta en los mundos virtuales hace factible la coordinación de diversas actividades grupales masivas. Aunado a la sensación del tiempo, los usuarios en los mundos virtuales experimentan nociones como el espacio, la distancia y el entorno.

La configuración en red que establecen los usuarios es otro rasgo constitutivo de los mundos virtuales en la Internet. Los participantes de los mundos virtuales tienen la capacidad de interactuar con los demás y con el entorno. La interacción con los demás establece lazos y eventualmente grupos de afiliación (Griol et al. 2019).

Aplicaciones como Discord se han caracterizado por ser espacios relacionales, en donde las personas han conseguido construir significados compartidos y una identidad social. De acuerdo con Herrera (2021), Discord es una plataforma que ofrece distintos canales de comunicación. Su gran acierto ha sido partir de un enfoque de construcción de comunidad, lo que posibilita a los usuarios entrar en contacto con otras personas con las que puede compartir gustos e intereses sobre cualquier tópico.

Finalmente, el soporte computacional es la última característica presente en estos mundos virtuales. De acuerdo con Bell (2008), los mundos virtuales en la Internet requieren de una infraestructura física, la cual hace posible la definición de las demás características que se le atribuyen a estos mundos. 
La consideración de estos rasgos definitorios, es de suma relevancia para poder plantear una posible delimitación de los mundos virtuales en la Red, pero en la búsqueda de las bases psicológicas, sociales y culturales de estos entornos, es necesario despojar el fenómeno de toda fundamentación tecnológica, lo que implica recuperar los planteamientos de Bittarelo (2014), quien expande la noción de mundo virtual al argumentar que lo sustantivo de estos entornos, no estriba en lo tecnológico, sino en la posibilidad de concebir espacios alternos al mundo concreto.

A partir de ello, se define un mundo virtual como un mundo imaginario sostenido principalmente por la dimensión narrativa subyacente, en el que los participantes pueden formar parte de la historia que se construye en el interior de estos entornos, y a través del uso activo de su imaginación, tienen la capacidad no sólo de ser espectadores, sino también de transformar el flujo de la historia o crearla.

Por lo tanto, su formulación significa considerar que los mundos virtuales anteceden a la Era Digital y se encuentran articulados en juegos de rol, en mitos, leyendas, historias, cuentos y creencias religiosas que forman parte de la cultura.

\subsection{El papel de los artefactos en la constitución de mundos virtuales}

La importancia de considerar la existencia de mundos virtuales antes de la Era de la Internet, implica suponer que la presencia de esta clase de entornos simbólicos requiere algo más que el soporte computacional. A partir de lo anterior, es posible pensar que los mundos virtuales pueden prescindir de una infraestructura de telecomunicaciones; sin embargo, dichos mundos no pueden omitir el papel de una infraestructura simbólica compuesta de distintos artefactos culturales.

La noción de artefacto cultural permite describir la relación estructural entre el individuo y el entorno, la cual es mediada a través de instrumentos que se configuran desde lo social y lo cultural. Para Cole (1996), un artefacto es un aspecto del mundo material o simbólico que se ha modificado a lo largo de la historia y se va incorporando al acervo cultural. Un ejemplo de un artefacto material puede ser un cuchillo que se ha transformado de acuerdo con las metas planteadas socialmente. Por otra parte, el lenguaje es el ejemplo por excelencia de un artefacto inmaterial que forma parte de la cultura y es apropiado por el individuo.

Para el desarrollo de esta disertación, se plantea que los mundos virtuales se constituyen por tres clases de artefactos inmateriales. El primero refiere a aquellos artefactos involucrados en la generación de mundos virtuales. La importancia de este primer punto de análisis estriba en que, como se mencionó, una característica de los mundos virtuales es que se trata de espacios alternos a la realidad concreta. Al no formar parte de la realidad física, 
es importante rastrear el origen de dichos mundos, respondiendo a la pregunta ¿cuál es el principio fundamental que permite la concepción de mundos nuevos?

El segundo consiste en los artefactos inherentes a la experimentación de habitar un entorno simbólico. Como se expresó anteriormente, un mundo es un espacio que se puede habitar; sin embargo, la realidad virtual no cuenta con un espacio tridimensional en el que las personas se pueden desplazar a voluntad; en su lugar, los mundos virtuales poseen un espacio abstracto que es habitable a través de lo que se conoce genéricamente como la mente humana.

La última dimensión refiere al lenguaje. La razón de este planteamiento reside en dos cuestiones: la primera, al tratarse de mundos que no existen de manera concreta, sino a través de representaciones simbólicas, es importante conocer ¿cuál es el principal sistema de mediación que sustenta la existencia de estos mundos? La segunda: el papel claramente social de estos espacios hace necesaria la prevalencia de sistemas de comunicación y construcción de significados.

A partir de estos tres puntos, se pretende analizar el papel de los artefactos que dan sustento y cabida a los mundos virtuales, partiendo de la consideración de aquellos mundos que no requieren de un soporte informático para su existencia.

\subsection{La creatividad como un artefacto para la creación de nuevos mundos}

Glăveanu (2010) indica que la representación del talento creativo es tan antigua como la civilización misma. Es posible ver estas alusiones en la cultura griega y romana, en donde el creador se vinculaba con la inspiración divina.

Los antiguos griegos utilizaban la palabra poein para referirse a aquella práctica en la que los pensadores, convertían sus ideas en palabras estéticamente correctas (Álvarez et al. 2017). Aunque esta cultura no tuviera un concepto propiamente claro sobre la creatividad, el poeta o su poesía contenían esos atributos imprescindibles de dicho fenómeno.

En la actualidad, la creatividad comenzó a ser de interés en el campo científico a mitad del siglo XX, cuando el presidente en turno de la American Psychological Association hizo notar en un discurso, que el fenómeno de la creatividad había sido descuidado por muchos años por los psicólogos de aquella época (Guilford, 1950).

Desde entonces, se exacerbó la investigación relacionada con este constructo, pero ¿cuál es la relación que guarda la creatividad con los mundos virtuales? Para ello, hay que partir de la definición de la creatividad. Una de las definiciones más elementales es la que proporciona el diccionario Merriam-Webster (s.f), el cual menciona que la palabra "creatividad" refiere a la acción de "crear" que significa to bring into existence (traer a la existencia). 
Esta primera acepción es de gran importancia, ya que, si la virtualidad refiere a una realidad alternativa a la realidad concreta, la creatividad es una capacidad humana que permite traer a la existencia nuevas realidades, aunque éstas en ocasiones sólo intenten replicar a la realidad concreta. En los mundos virtuales, como en el caso de los relatos mitológicos, el proceso creativo es inherente, porque a través de estos mundos, distintas civilizaciones son capaces de concebir realidades que están compuestas de seres mágicos, con cualidades peculiares, lugares extraordinarios con un funcionamiento y una lógica endémica, propia de esa realidad en particular.

Como menciona Bittarelo (2014), la creación y perduración de dichos mundos tiene una dimensión claramente social, debido a que éstos son más que el resultado de una contribución individual. La autoría anónima del mito refleja la distribución social con la que se crea el relato, el cual integra las diferentes aportaciones individuales y las consolida a través del tiempo como un artefacto cultural.

Dada la naturaleza social de los mundos virtuales, se requieren planteamientos que contemplen tanto los factores psicológicos, sociales y culturales que intervienen en la creación y postergación de los mundos virtuales, dado que éstos son el resultado de la interacción de los individuos con su contexto sociocultural.

El modelo de sistemas de la creatividad propuesto por Csikszentmihalyi (2015) es de gran utilidad para comprender los procesos creativos subyacentes a los mundos virtuales. Desde su posicionamiento, este autor sustenta que la creatividad es el resultado de tres dimensiones o sistemas: (1) el campo: que refiere al conjunto de instituciones sociales que selecciona aquellas producciones que vale la pena preservar; (2) el dominio cultural: que contiene las reglas simbólicas de acción y posibilita la transmisión de ideas a próximas generaciones; y por último (3) el individuo: quien es el que produce algún cambio o variación en el dominio.

En lo referente a la creación, transformación y postergación de los mundos virtuales, dicho modelo resulta útil para poder comprender los procesos tanto a nivel psicológico, social y cultural. De manera ilustrativa, se tomará el mito de la Atlántida, la cual era una isla caracterizada por su capacidad militar. Este mítico lugar estaba rodeado por montañas y las construcciones que la conformaban se caracterizaban por su enorme tamaño, debido a que esta ciudad pasaba por una época de prosperidad (Bittarelo, 2014).

El relato sobre la Atlántida aparece descrito por primera vez en los diálogos de Platón conocidos como Timeo y Critias. Según estos escritos, la Atlántida era una tierra perteneciente al dios griego Poseidón, quien se enamoró de una humana con la que tuvo diez hijos. Poseidón decidió dividir la isla en diez segmentos de tal manera que su descendencia tuviera un lugar sobre el cual reinar. Al mayor de sus hijos, llamado Atlas, le entregó la tierra 
más preciada del lugar y, además, Poseidón lo envistió de autoridad sobre sus hermanos, de aquí que el nombre de la isla fuera la Atlántida (Ramage, 1978).

Ramage (1978) señala que los diálogos de Platón, Timeo y Critias, son las fuentes oficiales en las que apareció por primera vez el mito de la Atlántida. Lo anterior invita a creer que dicho filósofo griego fue el creador de tan increíble mundo virtual, pero en realidad no es así. Platón deja ver que la historia forma parte del acervo cultural de los atenienses antiguos. El texto de Critias expresa claramente que esta historia fue el resultado de la transmisión y transformación de numerosas generaciones de atenienses.

Al respecto Csikszentmihalyi (2015) señala que lo que se considera una creación individual es realmente el resultado de procesos sociales relacionados con la negociación y la legitimización. La delimitación de dónde empieza el proceso creativo, es completamente arbitraria. Por lo regular se suele pensar que el proceso comienza con la persona creadora, pues es en él donde comienza la idea innovadora. Sin embargo, el autor señala que la aportación individual se sustenta de las prácticas habituales, el lenguaje, las creencias, las costumbres; en otras palabras, de la cultura.

Los sistemas involucrados en el acto creativo están presentes en el mito de la Atlántida. El sistema del campo está compuesto por aquellos grupos de personas e instituciones sociales que postergaron el relato. La Academia fundada por Platón es un ejemplo de estas instituciones atenienses, la cual tenía como propósito el análisis profundo del conocimiento humano (Franyutti, 2019). Gracias a este sistema es posible que los relatos trasciendan en el tiempo, pues el campo es el encargado de preservar el mundo virtual y de legitimar los nuevos fragmentos que se añaden a lo largo del tiempo.

El sistema del dominio está presente en la cultura griega politeísta, que se caracterizaba por representar a sus deidades con formas humanoides, pues las principales virtudes que los griegos apreciaban eran la juventud y la belleza. La antigua cultura griega se caracterizó por una tendencia a relatar historias en las que sus deidades protagonizaban situaciones extraordinarias que les permitían explicar la naturaleza de su realidad (Kindt, 2012).

Finalmente, el campo del individuo queda representado por las aportaciones creativas que hace cada persona, como lo refleja Platón. Sin embargo, como menciona Csikszentmihalyi (2015) no todas las aportaciones individuales son incorporadas al acervo cultural (el sistema del dominio), pues son las instituciones sociales las que legitiman qué fragmentos del relato se incorporan y cuáles otros se descartan. Lo anterior refleja la interacción intrínseca entre los tres sistemas descritos. 
En síntesis, los mundos virtuales implican espacios que existen de manera alterna y por lo tanto su concepción requiere forzosamente de un acto creativo, el cual se define como una capacidad humana que posibilita la creación de nuevas realidades. La naturaleza social en la creación y perduración de los mundos virtuales implica concebir el proceso creativo no como una acción individual, sino como una actividad distribuida socialmente que implica un complejo sistema de interacciones que involucra la dimensión social, cultural e individual.

\subsection{La imaginación como artefacto para habitar mundos virtuales}

Los mundos virtuales se caracterizan por ser espacios con un ambiente, una función, una estética y un orden que hacen posible poder habitarlos (Tosello, 2012). La palabra habitar, suele usarse para referirse a prácticas cotidianas en las que cada individuo construye significados, representaciones e imágenes sobre los espacios y lugares en los que se desenvuelve (Cireddu y Díaz, 2016).

La carga subjetiva que se atribuye a los espacios, es la manera en la que el sujeto logra apropiarse de ellos. Al respecto Lefebvre (1971 en Bazán y Siedl, 2011) plantea que habitar significa llevar a cabo acciones que le permiten a un individuo apropiarse de algo, en donde la apropiación no apela a la propiedad, sino a la intención de convertir algún fragmento de la realidad en una manifestación de su subjetividad.

La definición que sugiere Lefebvre es lo suficientemente amplia como para despojar al término de las concepciones coloquiales que lo relacionan con la posibilidad de ocupar espacios físicos. Apelando a la postura de dicho filósofo, se vuelve factible poder habitar objetos, historias, costumbres y espacios simbólicos como lo son los mundos virtuales.

La falta de materialidad de los mundos virtuales, motiva al ser humano a usar habilidades pautadas culturalmente que le permiten habitar espacios etéreos. Una de estas habilidades es la imaginación, que puede ser definida como la capacidad de trascender mentalmente el tiempo y el espacio, para pensar en situaciones ficticias (Reese, 2013).

Tanto la creatividad como la imaginación están vinculadas a la creación o transformación novedosa de la realidad, pero la principal diferencia entre ambos constructos, estriba en que la imaginación implica un esfuerzo mental por representar algo que no está presente, mientras que la creatividad está relacionada con la posibilidad de traer a la existencia algo novedoso o transformado (Robinson y Aronica, 2009). De esta manera, un acto creativo puede incluir a la imaginación como una herramienta cognitiva en la creación de productos novedosos, pero la imaginación no necesariamente implica una acción creativa.

En relación con los mundos virtuales, la creatividad es un elemento sustancial que posibilita la concepción de mundos fantásticos con características únicas, mientras que la imaginación es un atributo que posibilita la representación mental de dichos mundos, pues 
los mundos virtuales sin soporte digital carecen de representaciones por sí mismas. En los mundos virtuales, como los relatos míticos, el individuo habita los espacios contados en la historia valiéndose de la representación mental que puede construir internamente.

Muchos mundos virtuales están referidos en la literatura. John Ronald Reuel Tolkien es autor de una novela de fantasía épica que lleva por nombre "El señor de los anillos". La historia describe un mundo poblado por seres ficticios tales como elfos, orcos, magos y otros seres mitológicos, que coexisten en un mundo virtual en el que interactúan persiguiendo sus propios intereses. Con el propósito de proveer a la historia de mayor realismo, el autor describe minuciosamente escenarios extraordinarios rodeados de montañas voluntariosas y bosques errantes (Pardo, 2012).

Mientras resulta innegable el peso creativo detrás de la concepción de mundos virtuales materializados en la literatura, la imaginación tiene un papel importante para el lector, ya que es a través de este atributo que es posible representar mentalmente lo que el autor describe a través del lenguaje escrito.

\subsection{El lenguaje como mediación en los mundos virtuales}

En los mundos virtuales, al tratarse de espacios intangibles, el individuo requiere de artefactos que le permitan operar sobre ellos. Uno de los artefactos inmateriales más importantes es el lenguaje, que se caracteriza por ser un complejo sistema de representación de objetos, acciones y relaciones. El lenguaje representa un importante puente entre el conocimiento desarrollado social e históricamente y los procesos cognitivos del individuo (Luria, 1976).

El lenguaje representa el principal artefacto cultural que da sostén a los mundos virtuales antes de la Internet, debido a que estos se articulan en el relato; la historia de lo acontecido. Por tanto, los primeros mundos virtuales se basaron en el lenguaje oral como principal medio de representación. La versatilidad de lenguaje fue fundamental para poder representar aquellos componentes extraordinarios que definen a las historias y que resultan inconcebibles en la realidad concreta.

Sólo a través del lenguaje es posible articular historias en las que, los mundos que se crean, no tienen un referente físico propiamente. Un ejemplo se puede encontrar en diferentes juegos de rol como Calabozos y Dragones, en el que los jugadores adoptan las características de un personaje, los cuales van tomando decisiones que modifican el flujo de la historia en el juego. En esta clase de mundos virtuales, uno de los jugadores se asume como el director del juego, quien tiene como función establecer el escenario en el que los demás jugadores están involucrados. Más allá del tablero y las piezas necesarias para el desarrollo del juego, 
éste se basa en la narrativa como una herramienta central sobre la cual, la historia se desenvuelve (Jiménez, 2020).

La naturaleza simbólica y social de los mundos virtuales, requiere forzosamente de sistemas de mediación que permitan la interacción de los individuos dentro de estos entornos. Quizá el principal artefacto para este propósito es el lenguaje, debido a que se trata de un sistema que facilita la comunicación e influencia recíproca entre las personas, así como la posibilidad de representar simbólicamente mundos que no tienen un referente concreto, como es el caso de los mundos virtuales.

Lo desarrollado hasta este punto permite vislumbrar que los mundos virtuales, al tratarse de realidades simbólicas que existen de manera paralela a la realidad concreta, logran prescindir del soporte computacional, pues estos mundos se manifiestan también en otras prácticas narrativas del ser humano, tales como los mitos, las historias, los juegos de rol, etcétera. Limpiar el concepto de la connotación tecnológica, ha permitido apreciar que la base fundamental de estos entornos estriba en diferentes artefactos culturales que favorecen la creación, la mediación y la habitabilidad de mundos virtuales.

\subsection{La prevalencia de los artefactos simbólicos en los mundos virtuales de la}

\section{Internet}

En la actualidad, la aparición de los mundos virtuales en la Internet ha hecho posible que algunos fenómenos que eran mediados por ciertos artefactos culturales, sean complementados por mediaciones digitales, por ejemplo, el desarrollo de la informática, la simulación y el modelado en 3D, ha hecho posible que los mundos virtuales en la Internet, dejen de ser referidos exclusivamente a través del lenguaje para ser representados también gráficamente. No obstante, muchos principios se mantienen vigentes, pero adaptados a los nuevos escenarios que plantea la Internet.

En lo que respecta a la concepción de nuevos mundos en el ciberespacio, la creatividad sigue siendo una capacidad necesaria para dicho propósito. Sin embargo, el proceso creativo se transforma, pues mientras que, en los mundos virtuales sin mediación tecnológica, grupos reducidos de personas son los encargados de asimilar y perdurar las diferentes aportaciones creativas que se conciben, en los mundos virtuales en la Internet la asimilación y perduración del aporte creativo se vuelve más horizontal.

Un claro ejemplo es Minecraft, el cual es un mundo virtual tridimensional en el que los usuarios pueden desplazarse en el entorno por medio de un avatar. La característica más distintiva de este videojuego es la capacidad que le brinda a sus usuarios de crear objetos virtuales tridimensionales (Pérez et al. 2017). 
Esta característica hace posible que los usuarios sean participantes activos en la creación del mundo virtual que representa Minecraft. Aunado a ello, los recursos virtuales ilimitados permiten que los escenarios concebidos sean tan extensos como la creatividad de los usuarios se los permita.

En relación con la habitabilidad de los entornos virtuales, los diferentes avances tecnológicos que se han suscitado en los últimos tiempos, han hecho que el uso de la imaginación como un recurso para la representación de espacios inaprensibles, haya sido desplazada por la representación gráfica.

No obstante, como bien lo señalan Cireddu y Díaz (2016), habitar representa la construcción de significados que el individuo realiza sobre el espacio (físico o simbólico). En los mundos virtuales en la Internet, este proceso ocurre a nivel psicosocial, a través de la manipulación simbólica que el individuo realiza sobre el entorno virtual, en donde la identidad cobra un papel importante.

La identidad es un complejo proceso dialéctico, en el que el individuo reconstruye su percepción del yo a partir de los rasgos identitarios que retoma activamente de los diferentes grupos sociales, los cuales a su vez están conformados por la contribución que realiza cada sujeto a la identidad social (Silva et al. 2018).

En lo que concierne a los mundos virtuales, la identidad representa aquel mecanismo psicosocial, en el que los usuarios construyen colectivamente significados que les permiten apropiarse del espacio simbólico, de tal forma que los participantes son capaces de sentirse inmersos en una realidad virtual, sin la necesidad de vislumbrar completamente una experiencia sensorial.

La identidad representa el vehículo por medio del cual los usuarios en la Red construyen significados compartidos que hacen de la experiencia virtual, una experiencia real, pero el fenómeno de la identidad no permite encontrar la razón por la que los usuarios de mundos virtuales como Minecraft, se sienten motivados no sólo a habitar estos espacios, sino también a ser agentes de cambio, transformación y recreación. Es posible encontrar en las ideas de Papacharissi (2015) una conceptualización que dé cuenta de ello.

El autor introduce la noción de Affective publics para enfatizar la manera en la que las emociones representan una fuerza que promueve el compromiso compartido dentro de la Internet. Los Affective publics encarnan el ingrediente que permite a los internautas pasar de una audiencia pasiva, a ser miembros activos que contribuyen directamente en las narrativas sociales. Dentro de los mundos virtuales, la presencia de "sentimientos compartidos" establece, no sólo los medios de identificación con el otro, sino también las motivaciones que posibilitan el mantenimiento de las prácticas que emergen dentro de los mundos virtuales. 
Por lo tanto, Affective publics promueve la creación y recreación de los mundos virtuales, así como las narrativas que en ellos subyacen.

En los mundos virtuales de la Internet, el lenguaje sigue siendo un principal instrumento de mediación. Como se mencionó, una característica de estos entornos es la interacción social que se establece gracias a la interconectividad. Garena Free Fire es un juego móvil de acción-aventura del género Battle royale, lanzado en diciembre de 2017, en donde el objetivo es sobrevivir en una isla remota con otros jugadores en línea que persiguen el mismo propósito. En esta plataforma, los participantes intercambian mensajes a través de salas de conversación y chats públicos o privados. Dentro de las posibilidades de comunicación, los usuarios pueden intercambiar textos, imágenes o audios (Rhoton, 2020).

El lenguaje, como sistema de símbolos, subyace a la interacción bajo cualquier canal de comunicación en los mundos virtuales. Sea por medio de conversaciones de texto, grabaciones de audio, videoconferencias, etcétera, el lenguaje posibilita la comunicación entre los usuarios, quienes comparten y negocian significados propios de la cultura que se gesta en los mundos virtuales.

World of Warcraft es un mundo virtual en la Internet, en donde se puede apreciar la enorme importancia que el leguaje tiene como principal instrumento de construcción social de significados, los cuales responden a las características contextuales específicas de dicho universo. Los usuarios han construido y consensuado una serie de términos para representar diferentes situaciones, acciones, objetos o personajes propios de este mundo.

En un foro de Internet, Alvinz (2010) recopila las diferentes palabras que se utilizan dentro de World of Warcraft, las cuales son el resultado de la experiencia compartida de los usuarios y el consenso implícito que se establece entre ellos. Por ejemplo, la palabra Buffs alude a aquellos hechizos o inyecciones que se utilizan para aumentar algún atributo del jugador, como la vida, la fuerza, la velocidad o el poder de la armadura. Esta clase de terminología deja ver que, la creación de nuevas realidades, requiere forzosamente una construcción social de símbolos y significados que permitan referirse a éstas. De aquí que, las tecnologías informáticas que soportan los mundos virtuales, sólo son la capa superficial de los componentes fundamentales de estos entornos.

\subsection{Los mundos virtuales como espacios para la socialización}

Para algunos investigadores, la aparición de espacios virtuales mediados por la Internet, representa una transformación cualitativa en la comunicación contemporánea, dado que la posibilidad de interconectividad permite a los usuarios establecer vínculos con personas a escala universal, lo que significa que amplifica la capacidad de interacción con otros, aunque no haya un encuentro presencial (Nagua et al. 2019). 
Es posible observar en diferentes espacios virtuales un sinnúmero de casos en los que las personas logran entablar distintas clases de relaciones. Ello se debe principalmente a la posibilidad que brinda la mediación digital para que las personas puedan mostrarse de manera espontánea, intercambiar ideas, sentimientos, valores, etcétera, lo que promueve el establecimiento y la consolidación de relaciones interpersonales. En consonancia con Bastidas (2020), los mundos virtuales se caracterizan por ser espacios para la socialización, en donde las personas se configuran en comunidades que poseen sus propios mecanismos de membresía y organización, por lo que los mundos virtuales pueden ser considerados como entornos para la interacción social.

No obstante, un análisis más crítico, hace evidente que la comunicación digital ha transformado los aspectos más fundamentales de esta clase de relaciones. Paradójicamente, los entornos virtuales si bien, han posibilitado que las personas estén más conectadas que en cualquier otro momento, también han hecho más efímeras estas conexiones. La efervescencia de la interacción en la Internet ha propiciado que los usuarios se inclinen por relaciones en donde la superficialidad es la constante.

Al respecto, Cornejo y Tapia (2011) plantean que las personas prefieren hablar de "estar conectado", en lugar de "conectar" con los demás. En lugar de hablar de "relaciones" los internautas prefieren aludir a "redes". Estos pequeños giros semánticos, tienen implicaciones profundas. Por ejemplo, a diferencia de las relaciones como el parentesco o el noviazgo, la idea de red representa el descompromiso voluntario con los demás en la virtualidad. Esto podría agudizar el empobrecimiento de las herramientas sociales con las que cuentan las personas para mantener una relación y afrontar los problemas que en ella emergen.

\section{Conclusiones}

La consolidación que la Internet ha tenido como una de las principales herramientas que facilitan la interacción entre personas, ha conllevado al análisis de fenómenos emergentes observados desde diferentes perspectivas. El desarrollo en distintas áreas aplicadas de la ciencia, como la informática, los sistemas de comunicación, los avances en la simulación, etcétera, han hecho posible la concepción de nuevos mundos caracterizados por conectar a personas ubicadas en diferentes latitudes del planeta, por medio de dispositivos computacionales que les permiten interactuar con el entorno digital y con otros usuarios conectados.

Estos mundos virtuales se diferencian de otra clase de escenarios por la autonomía que consiguen al prescindir del usuario, es decir, se trata de espacios persistentes que siguen existiendo independientemente de las decisiones individuales de cada internauta. No 
obstante, los mundos virtuales en la Internet, son sólo una variación del basto espectro de mundos simbólicos, en donde ciertos artefactos inmateriales son fundamentales para su existencia.

Este ensayo tuvo como objetivo demostrar a través de una gran variedad de casos documentados, que los mundos virtuales pueden prescindir de una infraestructura material, sin embargo, dichos mundos no pueden omitir el papel de artefactos como la creatividad, la imaginación, la identidad, las emociones, las relaciones interpersonales y el lenguaje.

Hablar de la importancia de la infraestructura simbólica de los mundos virtuales, tiene implicaciones tanto en el terreno teórico como en el práctico. Por un lado, esboza la posibilidad de replantear las concepciones predominantes que enfatizan la dimensión computacional en su delimitación. Esto representa un reto importante en la búsqueda de criterios que sean capaces de contener escenarios tan distintos como los que se encuentran en la Internet, así como aquellos que se entraman en narrativas culturales y se materializan en historias, leyendas, juegos de rol, etcétera.

De igual manera, el planteamiento presentado en este ensayo, invita al desarrollo de perspectivas transdisciplinarias que permitan la comprensión sistémica de los entornos virtuales, en donde se reconozcan aquellas analogías que el mundo digital guarda con el mundo físico, pero también que den claridad sobre los aspectos endémicos de las interacciones en los entornos de la Internet.

En el campo práctico, las nociones expuestas representan un puente conceptual que conecta a estos entornos virtuales en Internet, con otras prácticas humanas que existen fuera de la Red. En el mundo de la programación, este puente conceptual puede ser una pauta que dé guía en la creación de nuevos mundos virtuales, que sean sensibles a la manera en la que las personas echan mano a distintos artefactos culturales, para hacer de la experiencia digital, una experiencia cada vez más real.

Las vetas de análisis siguen siendo amplias, es importante continuar con reflexiones más profundas sobre cada uno de los artefactos culturales que son imprescindibles en la constitución de mundos virtuales, dado que el análisis presentado aquí dio prioridad a la amplitud sobre la profundidad. Es importante realizar análisis comparativos de los mundos virtuales dentro de la Internet y fuera de ella, que permitan identificar las similitudes y las diferencias en la operatividad que tienen cada uno de los artefactos presentados. Los escrutinios deben considerar no sólo la dimensión discursiva o teórica, sino también incorporar la observación sistemática soportada a partir de nuestras categorías analíticas.

Por último, estos fenómenos de naturaleza sociocultural, están presentes en todos los mundos virtuales; tanto en los mundos que se encuentran en el ciberespacio, como en 
aquellos que existen a través de distintos juegos de rol, mitos, leyendas, historias, etcétera. Los mundos virtuales son espacios que existen en una realidad abstracta a la que sólo se accede a través de mediaciones simbólicas, que se construyen, transforman y también perduran desde lo psicológico, lo social y lo cultural.

Este trabajo se ha financiado con una beca doctoral del Consejo Nacional de Ciencia y Tecnología (CONACYT) México, a través del Posgrado en Psicología de la Universidad Nacional Autónoma de México, el cual forma parte del Programa Nacional de Posgrados de Calidad (2018-000068-02NACF21376).

\section{Referencias bibliográficas}

Adams, P. C. (2014). Communication in Virtual Worlds. En M. Grimshaw (Ed.), Oxford handbook of virtuality (pp. 239-253). Oxford University Press.

Álvarez, J. B., Gómez, G. M., y Serrano, G. N. (2017). Hacer y saber hacer. Una propuesta al proceso formativo del arquitecto. Actas Congreso Iberoamericano redfundamentos, 1, 503-513.

Bastidas, K. A. (2020). Comunidad, interacción, membresía y videojuegos. Un análisis centrado en las formas de interacción digitales en el videojuego GTAV (Tesis). Universidad Central del Ecuador, Quito.

Bazán, C. I. y Siedl, A. C. J. (2011). Habitar el espacio. En III Congreso Internacional de Investigación y Práctica Profesional en Psicología XVIII Jornadas de Investigación Séptimo Encuentro de Investigadores en Psicología del MERCOSUR. Facultad de Psicología-Universidad de Buenos Aires.

Bell, M. W. (2008). Toward a definition of "virtual worlds". Journal For Virtual Worlds Research, 1(1).

Bittarelo, M. B. (2014). Mythologies of virtuality: "Other space" and "Shared dimension" from ancient myths to cyberspace. En M. Grimshaw (Ed.), Oxford handbook of virtuality (pp. 86-110). Oxford University Press.

Brey, P. (2014). The physical and social reality of virtual worlds. En M. Grimshaw (Ed.), Oxford handbook of virtuality (pp. 42-54). Oxford University Press.

Cireddu, A. y Díaz, V. (2016). Las urbanizaciones residenciales cerradas, una forma de habitar la ciudad contemporánea. En C. Alvarado, R. Gómez y R. Hidalgo (Eds.), 
Expresión territorial de la fragmentación y segregación (pp.31-52). Universidad Autónoma del Estado de Morelos.

Cole, M. (1996). Psicología cultural. Morata.

Cornejo, M. y Tapia, M. L. (2011). Redes sociales y relaciones interpersonales en internet. Fundamentos en humanidades, 12(24), 219-229.

Coyne, R. (2018). Transactions in virtual places: Sharing and excess in blockchain worlds. En E. Champion (ed.), The Phenomenology of Real and Virtual Places (pp. 76-93). Routledge.

Csikszentmihalyi, M. (2015). The systems model of creativity: The collected works of Mihaly Csikszentmihalyi. Springer.

Díaz, F. J. (2016). La evolución de Internet y las tecnologías móviles. Bit \& Byte, 2.

Franyutti, M. A. L. (2019). La influencia de las civilizaciones antiguas en la educación actual. Logos Boletín Científico de la Escuela Preparatoria No 2, 6(12), 12-13.

Garner, T. y Grimshaw, M. (2014). Sonic Virtuality Understanding Audio in a Virtual World. En M. Grimshaw (Ed.), Oxford handbook of virtuality (pp. 364-377). Oxford University Press.

Glăveanu, V. P. (2010). Paradigms in the study of creativity: Introducing the perspective of cultural psychology. New ideas in psychology, 28(1), 79-93.

Greenlaw, R. y Hepp, E. (2001). Inline/online: fundamentals of the internet and the world wide web. McGraw-Hill Higher Education.

Griol, D., Sanchis, A., Molina, J. M. y Callejas, Z. (2019). Developing enhanced conversational agents for social virtual worlds. Neurocomputing, 354, 27-40.

Guilford, J. P. (1950). Creativity. American Psychologist, 5(9), 444-454.

Jiménez, M. R. (2020). La narrativa experiencial como propuesta teórico-metodológica al campo de la comunicación y su aplicación en dos grupos de juego de Calabozos y Dragones. Global Media Journal México, 16(31), 105-124. https://doi.org/10.29105/gmjmx16.31-6

Kindt, J. (2012). Rethinking Greek Religion. Cambridge University Press.

Lehdonvitra, V. (2014). Virtuality in the Sphere of Economics. En M. Grimshaw (Ed.), Oxford handbook of virtuality (pp. 496-510). Oxford University Press.

Luria, A. R. (1976). Cognitive development: Its cultural and social foundations. Harvard university press. 
Martin, J. (2008). Consuming code: use-value, exchange-value, and the role of virtual goods in Second Life. Journal For Virtual Worlds Research, 1(2).

Minocha, S. y Hardy, C. (2016). Navigation and wayfinding in learning spaces in 3D virtual worlds. En S. Gregory, M. J. Lee, B. Dalgarno y B. Tynan (Eds.), Learning in virtual worlds: Research and applications (pp. 3-42). Athabasca University Press.

Nagua, D. X., Galarza, A. S. y Sare, F. I. (2019). Las redes sociales y las relaciones interpersonales de las nuevas generaciones de ecuatorianos. Conrado, 15(66), 31-37.

Narea, S. L. (2019). La Generación Millennials en un contexto multipantalla: viejas prácticas nuevos medios categoría salas de juego Fortnite. (Tesis). Universidad Casa Grande, Guayaquil.

Papacharissi, Z. (2015). Affective publics: Sentiment, technology, and politics. Oxford University Press.

Pérez, J. L. S., de la Torre Cantero, J., Dorta, N. M., Carrera, C. C. y Díaz, D. M. (2017). Creación e inserción de modelos 3D en Minecraft para la mejora de la competencia espacial y creativa en Ingeniería. In Imaginar y Comprender la Innovación en la Universidad: VII Jornadas de Innovación Educativa de la Universidad de la Laguna (pp. 303-312). Servicio de Publicaciones.

Ramage, E. S. (Ed.). (1978). Atlantis, Fact Or Fiction?. Indiana University Press.

Reese, E. (2013). Culture, Narrative, and Imagination. En M. Taylor (Ed.), The Oxford handbook of the development of imagination (pp. 196-211). Oxford University Press.

Robinson, K. y Aronica, L. (2009). The element: How finding your passion changes everything. Penguin.

Silva, M. I. C., Suárez, E. P. y Sierra, S. M. C. (2018). Redes sociales e identidad social. Aibi revista de investigación, administración e ingeniería, 70-77.

Snodgrass, J. G. (2016). Online virtual worlds as anthropological field sites: Ethnographic methods training via collaborative research of Internet gaming cultures. Annals of Anthropological Practice, 40(2), 134-147.

Sparrow, M. A. (2012). The law of virtual worlds and Internet social networks. Gower Publishing.

Timokhina, S. L. y Emelianenko, V. D. (2018). The anthropological aspect in the development of Internet's philosophy. Questions of student science, 10, 8-11. 
Tosello, M. E. (2012). La experiencia de habitar en Espacios Virtuales Interactivos. http://biblioteca.puntoedu.edu.ar/handle/2133/3924

Zhang, Y. G., Dang, Y. M., Brown, S. A. y Chen, H. (2017). Investigating the impacts of avatar gender, avatar age, and region theme on avatar physical activity in the virtual world. Computers in Human Behavior, 68, 378-387.

\section{Otros documentos consultados}

Alvinz (3, enero, 2010). Glosario del argot de World of Warcraft [Foro en la Web]. https://elforoblanco.foroactivo.com/t882-glosario-del-argot-de-world-of-warcraft

Castells, M. (2000, octubre). Internet y la sociedad red. Trabajo expuesto en la Conferencia de Presentación del Programa de Doctorado sobre la Sociedad de la Información y el Conocimiento, España.

Herrera, C. (10 de julio de 2021). El boom Discord: la app que pasó de los juegos a la gestión de equipos. La Nación. https://cutt.ly/WRZhXEF

McGill, A. (6 de febrero de 2008). Virtual World Continuum. Pew Research Center. https://www.pewresearch.org/internet/2008/02/06/virtual-world-continuum/

Merriam-Webster. (s.f.) Create. En Merriam-Webster.com dictionary. https://www.merriamwebster.com/dictionary/create

Pardo, C. G. (2012). El señor de los anillos [reseña bibliográfica]. https://cutt.ly/qRZhYu9

Rhoton, S. (Consultado junio, 2020). 10 trucos y consejos para jugar bien Free Fire [Foro en la Web]. https://www.ligadegamers.com/free-fire-consejos/ 